Surveillance

\section{Surveillance for rare disorders by the BPSU}

\section{Verity, M Preece}

\section{Why is it worthwhile?}

$T$ he British Paediatric Surveillance Unit (BPSU) was set up in 1986 and has involved paediatricians in a pioneering mechanism for the surveillance of rare but important conditions that affect the health of children. Judged by its output the Unit has been very successful. By the end of 2001, 49 projects had been completed, resulting in over 150 publications in peer reviewed journals. The aim of this article is to examine the reasons for the success of the BPSU and to consider whether or not the activities of the Unit are worthwhile.

\section{HOW DOES THE BPSU WORK?}

The basic idea behind the Unit is simple enough. ${ }^{1}$ Investigators who want to perform surveillance studies apply to the BPSU Executive Committee for places on the BPSU surveillance card. Each month the BPSU office sends the orange surveillance card to over 2000 consultant paediatricians in the United Kingdom and Eire. The card lists the disorders currently under surveillance. If a child with one of these disorders has been seen during the previous month, the local paediatrician is asked to tick the appropriate box on the card and return it to the BPSU office. The office then tells the relevant surveillance group so that the paediatrician can be contacted to obtain the clinical details, usually by questionnaire. Data about patients are held securely by the surveillance groups and no information about individual patients is held by the BPSU.

\section{WHY DOES THE BPSU WORK?}

\section{Support from key organisations}

The following were involved in planning the BPSU: the British Paediatric Association (now the Royal College of Paediatrics and Child Health), the Communicable Disease Surveillance Centre at Colindale, the Institute of Child Health (London), the Royal College of Physicians (Ireland), and the Scottish Centre for Infection and Environmental Health. Representatives from all these organisations-and others-have remained in active contact by attending the monthly meetings of the BPSU Executive Committee.
Active involvement of paediatricians Every month the card arrives in the in-tray, and each paediatrician makes a decision-do I bother to complete it or do I throw it in the bin? Fortunately in over $90 \%$ of cases the decision is made to complete the card and return it to the BPSU office. To maintain this interest it is important that paediatricians are not overloaded with BPSU requests. The conditions under surveillance have to be relatively rare and only about $20 \%$ of paediatricians report any case in a year. On most occasions all the paediatrician has to do is tick a "nothing to report" box. This "negative" feedback is very important because it enables Richard Lynn, the Scientific Coordinator, to keep track of response rates and investigate the reasons that cards fail to return. It also helps us to estimate the extent to which there is under reporting of cases. The BPSU Executive Committee also tries to avoid overloading paediatricians by encouraging surveillance groups to make their questionnaires simple and short.

\section{By choosing the right surveillance studies}

The conditions on the surveillance card must be sufficiently important for paediatricians to want to make the effort. When a paediatrician ticks a box on the orange card, this results in a request for further information, usually in the form of a questionnaire to complete. In itself this is hardly an incentive to report cases! It must be clear to paediatricians that each surveillance project is likely to yield information that will be beneficial to children in general.

\section{By saving effort}

Coordination of activities facilitates the collection of information. Clinicians are approached by one Unit and are not put off by receiving questionnaires from many different sources. Investigators are helped by the BPSU to formulate and organise projects.

\section{IS THE WORK OF THE BPSU WORTHWHILE?}

The two most important criteria for acceptance of a study are:
(1) That it attempts to answer a question of scientific importance

(2) That the outcome of the study is of clear relevance to public health.

It is interesting to review some BPSU studies in the light of these criteria. They fall into a number of distinct groups as shown below, although some do not fit neatly into one or other category.

\section{Infectious diseases}

One of the first BPSU studies was of haemolytic uraemic syndrome (HUS). This remains a topical subject-another study of the same condition has now been completed. The recent study showed that most cases of HUS in the UK are caused by verocytotoxin producing $E$ coli 0157, and in spite of the impression given by the newspapers most cases are sporadic-outbreaks are uncommon. ${ }^{2}$ As HUS surveillance has been undertaken by several other national surveillance units it has been possible to make international comparisons.

The BPSU has provided data to help decide whether or not to perform national screening programmes for congenital infections. It was found that there were sufficient numbers of cases of transmissible syphilis ${ }^{3}$ and of congenital rubella ${ }^{4}$ to justify continued screening. In contrast, other BPSU studies have shown that there are not sufficient numbers of cases of congenital toxoplasmosis $^{5}$ or neonatal herpes ${ }^{6}$ to justify national screening. More recently, clinician reporting by obstetricians through the Royal College of Obstetricians and Gynaecologists, supplemented by paediatric reporting via the BPSU and unlinked anonymous HIV surveillance, has shown that HIV is prevalent throughout the UK. ${ }^{7}$ It is estimated that routine universal voluntary antenatal HIV testing would be cost effective for London and possibly elsewhere.

In order to determine whether or not children are developing variant Creutzfeldt-Jakob disease (vCJD), the study of progressive intellectual and neurological deterioration (PIND) in children was devised. ${ }^{8}$ There is good evidence that vCJD results from human exposure to beef products that contain abnormal prion protein. However, there is no useful screening test for vCJD at present, and detection of cases in children is complicated by the fact that there are many rare childhood neurodegenerative disorders. The strategy is therefore to

Abbreviations: BPSU, British Paediatric Surveillance Unit; CJD, Creutzfeldt-Jakob disease; HUS, haemolytic uraemic syndrome; $M C A D$, medium chain acyl coenzyme $A$ dehydrogenase; PIND, progressive intellectual and neurological deterioration 
obtain clinical information about all children with PIND and present the anonymised details to an Expert Group of paediatric neurologists. The Expert Group carefully reviews the clinical picture and the investigations performed locally and classifies the cases according to diagnosis. After five years of surveillance 1320 children with suspected PIND have been reported to the study. It is relatively reassuring that just six children with VCJD have been identified in this age group, but they were all notified in the past three years. It is possible that more cases of vCJD will occur in children, so PIND surveillance continues. It would not be practical to perform this important public health surveillance without the active cooperation of paediatricians via the BPSU.

\section{Non-infectious conditions}

An example of a study of a relatively rare condition that had wide importance was that of medium chain acyl coenzyme A dehydrogenase (MCAD) deficiency, in which there is the possibility of a severe encephalopathy in susceptible children. ${ }^{9}$ This condition might cause death after an apparently minor illness, and this study highlighted the fact that MCAD deficiency could be wrongly classified as sudden infant death syndrome. Another important differential diagnosis is Reye's syndrome, which is a rare noninflammatory encephalopathy associated with hepatic dysfunction.

National surveillance for Reye's syndrome was transferred to the BPSU card in 1986 and was underway until 2001. This surveillance was able to document the dramatic reduction in incidence of "classic" Reye's syndrome after the Committee on Safety of Medicines issued warnings about the use of aspirin in children. ${ }^{10}$ The study of MCAD deficiency served to highlight the fact that a number of metabolic disorders may present with a "Reye like" illness that is clinically and pathologically similar to Reye's syndrome. Thus two BPSU studies shed light on each other and both helped to illuminate an important public health issue-the use of aspirin in young children.

\section{Common disorders that are relatively uncommon in childhood}

Big numbers (more than about 300 cases a year) overload the BPSU reporting system both for the paediatricians who fill in the questionnaires and the surveillance groups who gather the data. However, there have been two studies of the epidemiology of diabetes in children, ${ }^{112}$ and more recently studies of inflammatory bowel disease, ${ }^{13}$ blindness, ${ }^{14}$ and (currently) stroke. Investigators have been able to focus on important questions about these conditions that are peculiar to childhood.

\section{Physical and social surroundings} There have been studies of drowning ${ }^{15}$ and of fatal or severe reactions to food ingestion. ${ }^{16}$ There have also been three studies of various aspects of child abuse-Munchausen syndrome by proxy, ${ }^{17}$ subdural haemorrhage, ${ }^{18}$ and more recently, acute abdominal injury in the context of suspected child abuse. The BPSU has provided a means of coordinating and disseminating knowledge about these important and controversial areas of child care.

\section{Treatment}

In the past there have been two studies of haemolytic disease of the newborn, ${ }^{19} 20$ and a third is underway. These have been important because of the changing use of vitamin K prophylaxis in neonates. Because of the concern that injected vitamin K might predispose children to later malignancy, the routine parenteral use of this vitamin has changed and there are now several schedules for oral administration. The most recent study of vitamin $\mathrm{K}$ deficiency bleeding will be able to chart the effects of changes in management. Another example has been the study of cerebral oedema and death associated with diabetic ketoacidosis in children, ${ }^{21}$ and the possible relation between these outcomes and the treatment of children in diabetic coma.

\section{Vaccination}

In view of the high profile concern about the use of some vaccines in children, it is perhaps surprising that there have only been a couple of studies of problems related to vaccination. Part of the difficulty here is that vaccinations are given to whole populations and therefore the potential numbers for study are too large for the BPSU system. However, there has been a study of meningoencephalitis associated with measles, mumps, and rubella (MMR) vaccine. ${ }^{22}$ Furthermore, the study of acute flaccid paralysis was set up to ascertain whether or not polio had been eradicated from the UK. ${ }^{23}$ The BPSU has had meetings with the Medicines Control Agency, the proposal being that the BPSU system could be used to target particular drugs and possibly vaccines. This is an area of potential future development.

\section{CONCLUSION}

In the space available it is not possible to give more than a flavour of the many studies that have been carried out by the BPSU. It can be seen that BPSU studies have answered important scientific questions and have made a significant contribution to the health of children. Paediatricians in the United Kingdom and Eire are to be congratulated on all the work that they have put into completing the cards and questionnaires that make BPSU studies possible. Hopefully this review will inspire them to continue their active support for the work of their Surveillance Unit.

Arch Dis Child 2002;87:269-271

Authors' affiliations

C Verity, Addenbrookes Hospital, Cambridge, UK (ex-chair, BPSU Executive Committee)

M Preece, Institute of Child Health, London, UK (current chair, BPSU Executive Committee)

Correspondence to: Dr C M Verity, Child Development Centre, Box 107, Addenbrookes Hospital, Hills Rd, Cambridge CB2 2QQ, UK; christopher.verity@addenbrookes.nhs.uk

\section{REFERENCES}

1 Anon. How the surveillance system works. In: British Paediatric Surveillance Unit, 15th Annual Report. London: Royal College of Paediatrics and Child Health, 2001:5-8

2 Taylor CM, Milford DV, Adak GK, et al. Haemolytic uraemic syndrome (HUS). In: British Paediatric Surveillance Unit, 15th Annual Report. London: Royal College of Paediatrics and Child Health, 2001:19-21.

3 Hurtig A K, Nicoll A, Carne C, et al. Syphilis in pregnant women and their children in the United Kingdom: results from national clinician reporting surveys 1994-7. BM 1998;317:1617-19.

4 Tookey PA, Peckham CS. Surveillance of congenital rubella in Great Britain, 1971-96. BMU 1999;318:769-70.

5 Peckham CS, Logan S. Screening for toxoplasmosis during pregnancy. Arch Dis Child 1993;68:3-5.

6 Tookey P, Peckham CS. Neonatal herpes simplex virus infection in the British Isles. Paediatr Perinatal Epidemiol 1996;10:432-42.

7 Intercollegiate Working Party for Enhancing Voluntary Confidential HIV Testing in Pregnancy. Reducing mother to child transmission of HIV infection in the UK. London: Royal College of Paediatrics and Child Health, 1998

8 Verity CM, Nicoll A, Will RG, et al. Variant Creutzfeldt-Jakob disease in UK children: a national surveillance study. Lancet 2000;356: 1224-7

9 Pollitt RJ, Leonard JV. Prospective surveillance study of medium chain acyl-CoA dehydrogenase deficiency in the UK. Arch Dis Child 1998;79:116-19.

10 Hardie RM, Newton LH, Bruce JC, et al. The changing clinical pattern of Reye's syndrome 1982-90. Arch Dis Child 1996;74:400-5.

11 Metcalfe MA, Baum JD. Incidence of insulin dependent diabetes in children aged under 15 years in the British Isles during 1988. BM 1991;302:443-7.

12 Wadsworth E, Shield J, Hunt L, et al. Insulin dependent diabetes in children under 5 : incidence and ascertainment validation for 1992. BM 1995;310:700-3.

13 Sawczenko A, Sandhu BK, Logan RF, et al. Prospective survey of childhood inflammatory bowel disease in the British Isles. Lancet 2001;357: 1093-4.

14 Rahi J, Gilbert C, Russell Eggitt I, et al. Severe visual impairment and blindness. In: British Paediatric Surveillance Unit, 15th Annual Report. London: Royal College of Paediatrics and Child Health, 2001:30-1.

15 Kemp A, Sibert JR. Drowning and near drowning in children in the United Kingdom: lessons for prevention. BM 1992;304:1143-6.

16 Colver A, Cant A, MacDougall C. Fatal/severe allergic reaction to food ingestion. In: British Paediatric Surveillance Unit, 14th Annual Report. London: Royal College of Paediatrics and Child Health, 2000:19-21. 
17 McClure RJ, Davis PM, Meadow SR, et al. The epidemiology of Munchausen syndrome by proxy, non-acccidental drowning and non-accidental suffocation. Arch Dis Child 1996:75:57-61.

18 Hobbs C, Wynne J, Livingston J, et al. Subdural haematoma/effusion (SDH). In: British Paediatric Surveillance Unit, 14th Annual Report. London: Royal College of Paediatrics and Child Health, 2000:36-7.
19 McNinch AW, Tripp JH. Haemorrhagic disease of the newborn in the British Isles: two-year prospective study. BM 1991;303:1105-9.

20 McNinch AW, Tripp J. Vitamin K deficiency bleeding. In: British Paediatric Association Surveillance Unit, 9th Annual Report. London: British Paediatric Association, 1995:20-1.

21 Edge JA, Hawkins MM, Winter DL, et al. The risk and outcome of cerebral oedena developing during diabetic ketoacidosis Arch Dis Child 2001;85:16-22.

22 Maguire HC, Begg NT, Handford SG. Meningoencephalitis associated with MMR vaccine. Commun Dis Rep 1991;1:R60-1.

23 Salisbury DM, Ramsay ME, White JM, et al. Polio eradication: surveillance implications for the United Kingdom. J Infect Dis 1997;175(suppl I):S156-9

\section{POSTCARD FROM DOWN UNDER}

\section{No smoke without ads?}

$\mathrm{R}$ ading a British magazine imported into Australia I suddenly noticed the profusion of cigarette advertisements. Smoking advertising has been banned in Australia since the early nineties and its absence-like the resolution a headache or the departure of an unwelcome guest-is refreshing but soon forgotten.

The BMJ Publishing Group has a whole journal, Tobacco Control, devoted to smoking and related issues. I'd recommend anyone with a social conscience and a sense of humour to read their editorial following Nottingham University's acceptance of cash from British American Tobacco with which to establish a chair of International Business Ethics. ${ }^{1}$ Much research is published there and elsewhere, but I'd like to make a couple of observations from my time in Australia.

Firstly, it hasn't been immediately obvious to me that fewer people are smoking. In fact, visitors from the UK feel that there are more, especially in restaurants, which may simply be a feature of outdoor cafe society where the need to segregate smokers is less keenly demanded

Secondly, Australians seem less aware of when they were being exposed to cigarette advertising, although this may reflect my own (hyper aware) biases. My colleagues seem almost oblivious to cinema product placement and sponsorship of international sports like cricket and, if we believe the propaganda, the otherwise impoverished Formula 1 motor racing.

This leads to the third observation, which is that people seem unaware of product or brand-creep. Camel will always be a cigarette brand while they produce cigarettes, however trendy or well made the clothing. British American Racing will always be British American Tobacco, with the interests of their paymasters at heart. Kraft products will now always be, essentially, a front for Phillip Morris, to the extent where some people regard them in the same light as Nestlé products in the eighties.

These are no reasons to allow tobacco advertising to recommence, quite the contrary. But they are a reason to think more broadly about how to combat the tobacco industry. We should never forget that we are dealing with organisations which are fighting for their corporate lives-if not those of their customers-and that they know how to fight. In the past the interesting point has been made that negotiation with the tobacco lobby is a mistake; we should simply legislate since they will, ultimately, listen to nothing less. (And, demonstrably, sometimes not even that). If we are taking these issues seriously we should look at media literacy as our strongest weapon-teaching people to under-
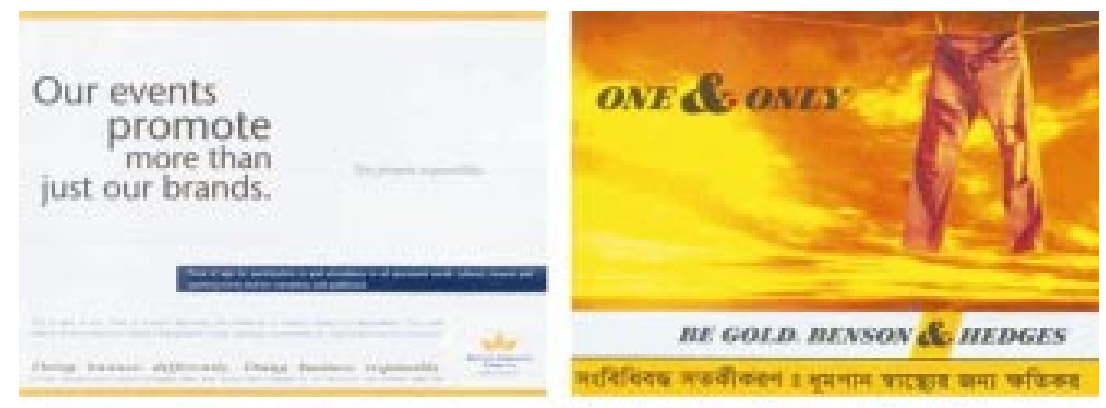

nE' GOR. MrVSOV AE MEDGES

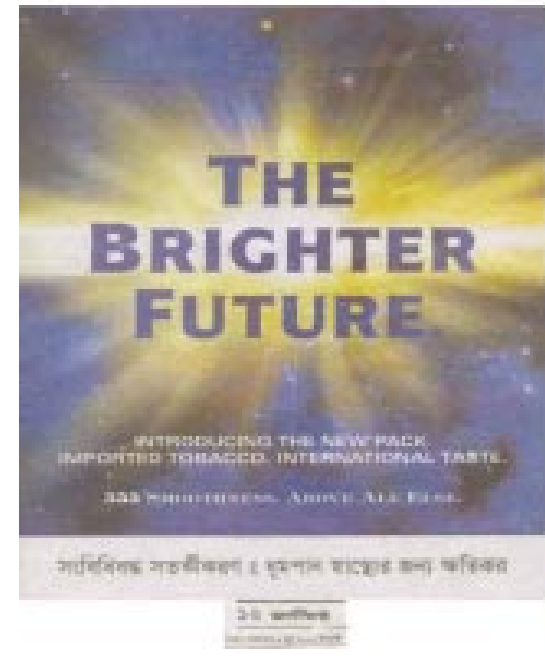

stand the subtext and motives behind advertisements, as distinct from the actual message.

We need also to bear in mind the action/ reaction dynamics in large multinationals. A third of a trillion dollar payout by the tobacco companies in the US is gratifying to us, and will hurt the industry, but it won't worry too much while it has unregulated access to 2.3 billion potential customers in India and China alone. It will just shift its market, and then up the price slowly.

Whatever data eventually emerge from the gradual banning of tobacco advertising in the non USA developed world, we should ignore the industry's fallacious arguments about advertising being aimed at brand shift rather than recruitment. It only takes a couple of neurones to do the sick maths the industry has done: if your product is killing 100000 of your customers prematurely a year in the UK alone, and you want to sell the same number of cigarettes each year, you need to recruit the same number each year. Preferably this will be from the young-teenagers and early twenties-because they are busy with the
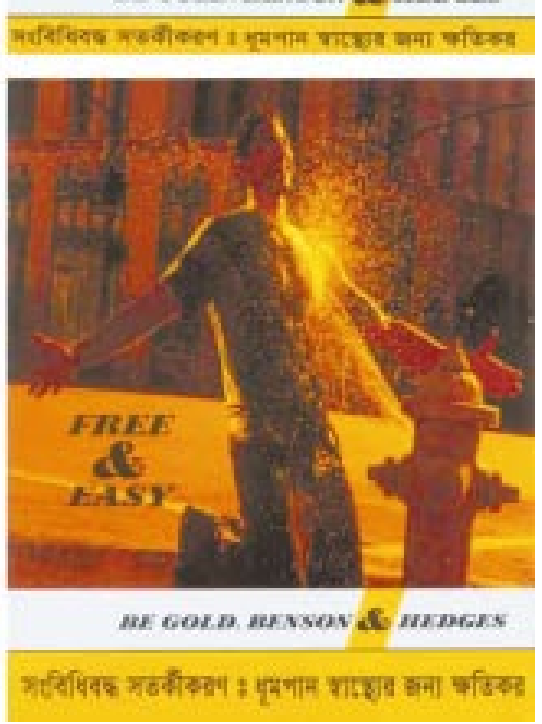

business of forging a life and an identity. If you can trick them that smoking says something positive about themselves, and keep them smoking for long enough to get them addicted, then you've replaced one of your 100000 . You just need to do that every 20 minutes, for ever.

The advertising ban is a victory, and may it last forever, but it is only a small one in the context of a long, protracted, and dirty international war which we are a long way from winning. We should congratulate ourselves, and then ask which of our children they are going to recruit in the next twenty minutes, and the twenty minutes after that,

I D Wacogne

Dr Wacogne was on secondment at the Royal Children's Hospital, Brisbane for two years and is now completing his SpR training at the North Staffordshire Hospital, UK.

1 Chapman S, Shatenstein S. The ethics of the cash register: taking tobacco research dollars. Tob Control 2001;10:1-2. and the twenty minutes after that.. 